\title{
Enhancement of the third-order nonlinear optical susceptibility in Si quantum wires
}

\author{
R. Chen \\ Department of Physics and Astronomy, State University of New York at Buffalo, \\ 239 Fronczak Hall, Amherst, New York 14260 \\ D. L. Lin \\ Department of Physics, Hong Kong University of Science and Technology, Clear Water Bay, Kowloon, Hong Kong \\ and Department of Physics and Astronomy, State University of New York at Buffalo, \\ 239 Fronczak Hall, Amherst, New York 14260* \\ B. Mendoza \\ Centro de Investigaciones en Optica, A. C. Apartado Postal 948, 37000 Leon, GTO, Mexico
}

(Received 15 April 1993)

\begin{abstract}
Recent observation of efficient light emission from porous silicon has attracted much attention and renewed interests in the study of nonlinear optical properties of nanometer-sized quantum systems. In this paper, we study the third-order nonlinear optical susceptibility of semiconductor quantum wires. The quantum wires are taken to be circular columns with a cross section size of $\sim 1 \mathrm{~nm}$. The excitonic effects are taken to be the major electronic excitations. We find that the quantum confinement of the excitons greatly enhances the third-order optical nonlinear susceptibility in a quantum wire. The source of the enhancement is primarily the confinement-induced localization of excitons. The large enhancement of the third-order optical nonlinearity estimated here is consistent with the recent observation of the efficient infrared-up-conversion luminescence in porous silicon.
\end{abstract}

\section{INTRODUCTION}

In the past few years, the nonlinear optical properties of semiconductor quantum wells and nanostructures have attracted much attention..$^{1-4}$ One of the most important features in these structures is that excitonic spectrum persists even at the room temperatures. ${ }^{5,6}$ This is due to the enhancement of the excitonic binding energy caused by the quantum confinement effect in these structures. It has been known that the effect of confinement sensitively depends on the relative size of the sample and the Bohr radius of the excitons in these structures. ${ }^{7,8}$

Recently, visible light luminescence from porous silicon (PS) has been observed. ${ }^{9}$ A great amount of research activities are devoted to a better understanding of the basic mechanism of efficient light emission from silicon nanostructures. A general consensus is that the quantum confinement in the free-standing nanometer silicon quantum wires formed by the chemical etching process is responsible for the efficient light emission. ${ }^{10} \mathrm{~A}$ blueshift in the absorption or emission spectrum as the porosity increases has been observed, ${ }^{11}$ which is characteristic of the quantum-size effects arising from the confinement of excitons to a volume smaller than that in the bulk materials. Further detailed studies ${ }^{12}$ of photoluminescence (PL) under different temperatures and various excitation intensities also support the idea that confined excitons may be involved in the radiative process. However, there are still considerable debates as to the precise origin of the visible luminescence in porous silicon. Possibilities other than confinement have been suggested. ${ }^{13-17}$

It has been shown primarily ${ }^{18-20}$ that the nonlinear op- tical polarizability may be greatly enhanced for an assembly of multiple-quantum-well structures and semiconductor microcrystallites, which have one- and threedimensional confinement, respectively. It is expected, therefore, that an optical nonlinearity enhancement of PS would exist if the hypothesis of quantum wires does indeed work. As a matter of fact, recent experimental observation $^{21}$ of efficient infrared-up-conversion luminescence in PS suggests a strong enhancement of the thirdorder nonlinear optical response. In this paper, we present a study of optical nonlinearity in Si quantum wires with a geometry of columns with a circular cross section. We find a large enhancement of optical nonlinearity in silicon nanometer-sized wires having a diameter of around $\sim 1 \mathrm{~nm}$. The source of the enhancement has been identified as quantum-confinement-induced localization of excitons in the quantum wires.

The paper is arranged as follows. In Sec. II we describe the electronic states in a quantum wire. The basic electronic excitations are taken to be excitonic states with a weak average Coulomb interaction between the electrons and holes. In Sec. III we study the oscillator strength of the basic optical transitions in the quantum wires. In Sec. IV the third-order optical susceptibility in quantum wires is calculated and the enhancement respect to the bulk $\mathrm{Si}$ is estimated. A brief summary is given in Sec. $\mathbf{V}$.

\section{ELECTRONIC EXCITATIONS IN QUANTUM WIRES}

There are several energies characteristic in quantum wires. The first are the size-quantization energies of the 
electrons and holes, denoted by $\Delta E_{c}$ and $\Delta E_{v}$, respectively. The second is the average Coulomb attraction between an electron and a hole in the quantum wire, $V_{\mathrm{ex}}$. The third is the interaction energy $V_{\text {int }}$ between two excitations when they are formed in the quantum wires. The exciton-exciton interaction will cause the excitons to derivate from an ideal boson. In silicon nanometer-sized wires, the quantum confinement greatly enhanced the exciton binding energy and makes the exciton binding energy much bigger than the size-quantization energy of the electrons or holes. In the following, we will assume the condition $V_{\text {exc }} \gg \Delta E_{c}, \Delta E_{v}$. The electronic excited state in a quantum wire can then be described as

$$
\Psi_{n}=\sum_{j} R_{n}(j) W_{j}^{c}\left(\mathbf{r}_{j}\right) \prod_{(i \neq j)} W_{i}^{v}\left(\mathbf{r}_{i}\right)
$$

where $W_{i}^{v}$ and $W_{j}^{c}$ are Wannier functions of the valence and the conduction bands, respectively, and $R_{n}(j)$ is the envelope function in a quantum wire of circular cross section, given as

$$
R_{n}(\rho)=\frac{\sqrt{2}}{R_{0}} \frac{J_{m}\left(k_{\|} \rho\right)}{J_{m+1}\left(k_{\|} R_{0}\right)} \frac{1}{\sqrt{2 \pi}} e^{i m \varphi} \frac{1}{\sqrt{L}} e^{i k_{z} z}
$$

where $\rho=u j, u$ is the unit-cell size, $j$ is the site index and its value is chosen from positive integers between 1 and $N, R_{0}$ is the radius of the wire $\left(R_{0}=u N\right)$, and $J_{m}$ is the Bessel function. The quantity $k_{\|}$is given by

$$
k_{\|}=\frac{\lambda_{m, n}}{R_{0}},
$$

where $\lambda_{m, n}$ is the zero of the Bessel function

$$
J_{m}\left(\lambda_{m, n}\right)=0
$$

The excitation energy spectrum of the conduction electrons can then be expressed as

$$
E=\frac{\hbar^{2} k_{z}^{2}}{2 m_{e}}+\frac{\hbar^{2}}{2 m_{e}}\left(\frac{\lambda_{m, n}}{R_{0}}\right)^{2} .
$$

The ground state of the system is

$$
\Psi_{g}=\prod_{i} W_{i}^{v}\left(\mathbf{r}_{i}\right)
$$

For the parabolic conduction and valence bands with effective mass $m_{e}$ and $m_{h}$, respectively, the size quantization is governed by the relationship among the sample size $R_{0}=N u$, and the effective Bohr radii of electrons $a_{e}=\hbar^{2} / m_{e} e^{2}$ and holes $a_{h}=\hbar^{2} / m_{h} e^{2}$. The sizequantization energies can be expressed for the electron and the hole, respectively, as follows:

$$
\begin{aligned}
& \Delta E_{c}=\left(\frac{\lambda_{m, n}}{R_{0}}\right)^{2} \frac{\hbar^{2}}{2 m_{e}}, \\
& \Delta E_{v}=\left(\frac{\lambda_{m, n}}{R_{0}}\right)^{2} \frac{\hbar^{2}}{2 m_{h}} .
\end{aligned}
$$

The exciton energy $V_{\text {exc }}$ is given by

$$
V_{\mathrm{exc}}=\frac{e^{2}}{\varepsilon_{0} a}=\frac{e^{2}}{\varepsilon_{0}\left(a_{3}+a_{h}\right)}
$$

where $a$ is the Bohr radius of the exciton. By taking into account the average Coulomb interaction between the electrons and holes, the excitation states in (1) should be modified to

$$
\Psi_{n}=\sum_{j} \sum_{j^{\prime}} R_{n}(\bar{j}) \phi\left(j_{r}\right) W_{j}^{c}\left(\mathbf{r}_{j}\right) \prod_{i \neq j} W_{i}^{v}\left(\mathbf{r}_{i}\right),
$$

where $R_{n}(\bar{j})$ is the envelope function given in (2), describing the center-of-mass motion of the exciton with $\bar{j}=\left(m_{e} j+m_{h} j^{\prime}\right) /\left(m_{e}+m_{h}\right)$, and $\phi\left(j_{r}\right)$ describes the electron-hole relative motion with $j_{r}=j-j^{\prime}$. For the lowest state of the exciton, the center-of-mass motion of the exciton can be written as

$$
\phi_{1 s}(r)=\left[\frac{1}{\pi a^{3}}\right]^{1 / 2} e^{-r / a},
$$

where $r=u\left(j-j^{\prime}\right)$, is the electron-hole separation.

\section{OSCILLATOR STRENGTH IN QUANTUM WIRES}

The transition dipole moment to the excited states $\Psi_{n}$ from the ground state $\Psi_{g}$ in the quantum wire can be evaluated as the following:

$$
\begin{aligned}
\left\langle\Psi_{n}|P| \Psi_{g}\right\rangle & =\sum_{j j^{\prime}} R_{n}(\bar{j}) \phi\left(j_{r}\right)\left\langle W_{j}^{c}\left(\mathbf{r}_{j}\right)\left|p_{j}\right| W_{j^{\prime}}^{v}\left(\mathbf{r}_{j^{\prime}}\right)\right\rangle \\
& =p_{c v} \phi_{1 s}(0) \int_{0}^{R_{0}} \rho d \rho \frac{\sqrt{2}}{R_{0}} \frac{J_{m}\left(k_{\|} \rho\right)}{J_{m}\left(k_{\|} R_{0}\right)} \int_{0}^{2 \pi} \frac{1}{\sqrt{2 \pi}} e^{i m \varphi} d \varphi \frac{1}{\sqrt{L}} \int_{0}^{L} e^{i k_{z}} d z,
\end{aligned}
$$

where $P$ is a compound of the dipole moment operator $\mathbf{P}$,

$$
\mathbf{P}=\sum_{i} \mathbf{p}_{i}
$$

and $\left\langle W_{j}^{c}\left(\mathbf{r}_{j}\right)\left|p_{j}\right| W_{j^{\prime}}^{v}\left(\mathbf{r}_{j^{\prime}}\right)\right\rangle=p_{c v} \delta_{j j^{\prime}}$. We see that the dipole matrix element will be zero unless $m=0$. Setting $m=0$, we have 


$$
\begin{aligned}
\left\langle\Psi_{n}|P| \Psi_{g}\right\rangle= & p_{c v} \phi_{1 s}(0)\left(\frac{2}{\sqrt{L}} \frac{\sin \left(\frac{k_{z} L}{2}\right)}{k_{z}}\right) \\
& \times \int_{0}^{R_{0}} \rho d \rho \frac{\sqrt{2}}{R_{0}} \frac{J_{0}\left(k_{\|} \rho\right)}{J_{1}\left(k_{\|} R_{0}\right)} \\
= & p_{c v} \phi_{1 s}(0) \frac{\sqrt{2}}{k_{\|}} \frac{2}{\sqrt{L}} \frac{\sin \left(\frac{k_{z} L}{2}\right)}{k_{z}} .
\end{aligned}
$$

Taking $k_{z} \rightarrow 0$, we have

$$
\left\langle\Psi_{n}|P| \Psi_{g}\right\rangle=p_{c v} \phi_{1 s}(0) \frac{\sqrt{2}}{k_{\|}} \sqrt{L} \quad\left(k_{z} \rightarrow 0\right),
$$

where $k_{\|}=\lambda_{0 n} / R_{0}$ and $J_{0}\left(\lambda_{0 n}\right)=0$.

The oscillator strength $f_{n}$ is given by

$$
f_{n}=\frac{2 m}{\hbar} \omega_{n}\left|p_{c v}\right|^{2}\left|\phi_{1 s}(0)\right|^{2} \frac{2 L R_{0}^{2}}{\lambda_{0 n}^{2}} \text {. }
$$

The oscillator strength per unit volume $\bar{f}_{n}$ can be obtained by multiplyirg (15) by a factor of $1 /\left(\pi R_{0}^{2} L\right)$, and is given by

$$
\bar{f}_{n}=\frac{4 m}{\pi \hbar} \omega_{n}\left|p_{c v}\right|^{2}\left|\phi_{1 s}(0)\right|^{2} \frac{1}{\lambda_{0 n}^{2}} .
$$

Notice that $\bar{f}_{n}$ is independent of the dimension of the quantum wire.

\section{THIRD-ORDER OPTICAL NONLINEARITY IN QUANTUM WIRES}

In this section we will study the third-order optical nonlinearity $\chi^{(3)}$ in quantum wires. The second-order optical nonlinearity would be zero in these structures due to the inversion symmetry. The third-order optical nonlinearity is not zero and we shall show that there is a large enhancement of $\chi^{(3)}$ in quantum wires of size $\sim 1 \mathrm{~nm}$. This enhancement originates from the quantum confinement effect of the excitons in the quantum wire.

The third-order optical polarizability $\chi^{(3)}$ in a quantum wire can be evaluated as the following: ${ }^{22}$

$\chi^{(3)}=\sum_{n \neq g} \sum_{n^{\prime}} \sum_{n^{\prime \prime} \neq g} \hbar^{-3} e^{4} x_{g n} x_{n n^{\prime}} x_{n^{\prime} n^{\prime \prime}} x_{n^{\prime \prime} g}[\cdots]$,

where $x_{n n^{\prime}}=\left\langle n|x| n^{\prime}\right\rangle$ and the terms in the brackets $[\cdots]$ are

$$
\begin{aligned}
{[\cdots]=} & \frac{1}{\left(3 \omega-\omega_{n^{\prime \prime} g}\right)\left(2 \omega-\omega_{n^{\prime} g}\right)\left(\omega-\omega_{n g}\right)}-\frac{1}{\left(3 \omega+\omega_{n^{\prime \prime} g}\right)\left(2 \omega+\omega_{n^{\prime} g}\right)\left(\omega+\omega_{n g}\right)} \\
& +\frac{1}{\left(\omega-\omega_{n^{\prime \prime} g}\right)\left(2 \omega-\omega_{n^{\prime} g}\right)\left(\omega+\omega_{n g}\right)}-\frac{1}{\left(\omega+\omega_{n^{\prime \prime} g}\right)\left(2 \omega+\omega_{n^{\prime} g}\right)\left(\omega+\omega_{n g}\right)} .
\end{aligned}
$$

Here we consider only the resonant case in which the energy of the incident photon $\hbar \omega$ is nearly equal to the first exciton energy $\hbar \omega_{0}$, and neglect contributions to the nonlinear polarization from the other levels. The nonzero contribution of the terms in Eq. (17) can be represented by the Feynman diagrams in Fig. 1. The contribution comes from two terms given by

$$
\begin{aligned}
& \langle 0|x| 1\rangle\langle 1|x| 0\rangle\langle 0|x| 1\rangle\langle 1|x| 0\rangle, \\
& \langle 0|x| 1\rangle\langle 1|x| 2\rangle\langle 2|x| 1\rangle\langle 1|x| 0\rangle .
\end{aligned}
$$

These correspond, respectively, to diagrams (a) and (b) in Fig. 1. Here we denote the ground state, the excited state with an excitation and with two excitations, respectively, by $|0\rangle,|1\rangle,|2\rangle$. The two terms in Eq. (19) can be evaluated separately as

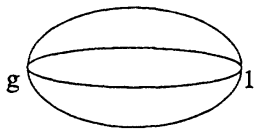

(a)

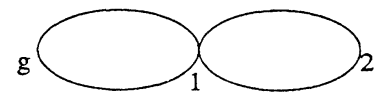

(b)
FIG. 1. The Feynman diagrams of the contributing terms in the $\chi^{(3)}$ expansion series. $e^{4}\langle 0|x| 1\rangle\langle 1|x| 0\rangle\langle 0|x| 1\rangle\langle 1|x| 0\rangle$

$$
=C \frac{\omega_{0}}{\left[\omega^{2}-\omega_{0}^{2}\right]\left[(3 \omega)^{2}-\omega_{0}^{2}\right]}
$$

and

$$
\begin{aligned}
e^{4}\langle 0|x| 1\rangle & \langle 1|x| 2\rangle\langle 2|x| 1\rangle\langle 1|x| 0\rangle \\
& =C \frac{\omega_{0}}{\left[\omega^{2}-\omega_{0}^{2}\right]\left[(3 \omega)^{2}-\omega_{0}^{2}\right]}\left(\frac{4 \omega^{2}}{\omega^{2}-\omega_{0}^{2}}\right),
\end{aligned}
$$

where

$$
C=\frac{16}{\hbar^{3}}\left|p_{c v}\right|^{4}\left|\phi_{1 s}(0)\right|^{4}\left(\frac{L^{2} R_{0}^{4}}{\lambda_{01}^{4}}\right) .
$$

Combining Eqs. (17), (19), (20), and (21) we get the following expression for $\chi^{(3)}$ :

$$
\chi^{(3)}=C \frac{\omega_{0}}{\left[\omega^{2}-\omega_{0}^{2}\right]\left[(3 \omega)^{2}-\omega_{0}^{2}\right]}\left[1+\frac{4 \omega^{2}}{\omega^{2}-\omega_{0}^{2}}\right] .
$$

Assume close-packed quantum wires; then the $\chi^{(3)}$ for the unit volume is given by 


$$
\begin{aligned}
\chi^{(3)}=\frac{16}{\pi \hbar^{3}}\left|p_{c v}\right|^{4}\left|\phi_{1 s}(0)\right|^{4} \frac{L R_{0}^{2}}{\lambda_{01}^{4}} & \\
& \times \frac{\omega_{0}}{\left[\omega^{2}-\omega_{0}^{2}\right]\left[(3 \omega)^{2}-\omega_{0}^{2}\right]}\left[1+\frac{4 \omega^{2}}{\omega^{2}-\omega_{0}^{2}}\right] .
\end{aligned}
$$

The large enhancement of $\chi^{(3)}$ comes from $\phi_{1 s}(0)$. Due to the exciton confinement in the quantum wire, the exciton size $a_{0}$ decreases with respect to the bulk value. From Eqs. (10) and (24) we see that the third-order nonlinear susceptibility sensitively depends on the Bohr radius $a_{0}$ as the inverse sixth power of $a_{0}, \chi^{(3)} \sim 1 / a_{0}^{6}$; a decrease in $a_{0}$ will lead to a large enhancement in $\chi^{(3)}$.

The effective of the exciton confinement in $\mathrm{Si}$ nanometer-sized wires may be attributed to an enhancement in the effective mass of electrons and holes. ${ }^{23}$ It has been known in quantum wells that there is a large enhancement in the effective mass of the electrons in the direction parallel to the well. ${ }^{24}$ Enhancement of electrons and hole effective masses is also expected to occur in quantum wires. According to realistic band-structure calculations done for nanometer-sized Si wires, ${ }^{25,26}$ we estimate a 2.5-time enhancement of the effective mass over the value in bulk $\mathrm{Si}$ in wires around $\sim 1 \mathrm{~nm}$ wide. This estimation is consistent with the temperature measurements of the exciton spectrum ${ }^{5,6}$ which are presented in terms of the binding-energy enhancement of the exciton. The enhancement of the effective mass will lead to a reduction in the Bohr radius by the same factor. If we denote the exciton Bohr radius in the bulk Si to be $a_{0 b}$, then $a_{0} / a_{0 b} \sim 1 / 2.5$. Therefore we estimate an enhancement in the third-order nonlinear susceptibility to be around $(2.5)^{6}=2.4 \times 10^{2}$, about two orders of magnitude higher than the bulk Si. Recently, effective infrared-upconversion luminescence in PS has been observed ${ }^{21}$ and the effects are attributed to a large enhancement in the third-order polarizability in PS. The order of magnitude of the enhancement estimated from the luminescence spectrum in Ref. 21 is consistent with the value obtained in this work.

\section{SUMMARY}

We present a simple and straightforward study of the third-order optical nonlinearity in nanometer-sized $\mathrm{Si}$ wires. We demonstrate that quantum confinementinduced exciton localization indeed leads to a large enhancement in $\chi^{(3)}$. For $\mathrm{Si}$ wires of size $\sim 1 \mathrm{~nm}$, the enhancement for $\chi^{(3)}$ is estimated to be around two orders of magnitude larger than that in the bulk Si. The third-order linearity is found to be very sensitively dependent on the exciton confinement. Under different conditions with large excitation density and reduced effectivemass enhancement, other factors, such as exciton-exciton interaction and size quantization of the electron and the hole states, can also contribute to nonlinearity in the quantum wire. A different source of enhancement of nonlinearity related to the dimension of the system has been put forward for quantum wells and microcrystallites. $^{18}$ A more elaborate study may be needed for a quantitative estimation of the optical nonlinearity under these conditions.
${ }^{*}$ Permanent address.

${ }^{1}$ D. S. Chemla and M. A. B. Miller, J. Opt. Soc. Am. B 2, 1155 (1985).

${ }^{2}$ P. Roussignorl, D. Ricard, K. C. Rustagi, and C. Flytzanis, Opt. Commun. 55, 143 (1985).

${ }^{3}$ R. K. Jain and R. C. Lind, J. Opt. Soc. Am. 73, 647 (1983).

${ }^{4}$ Optical Nonlinearities and Instability in Semiconductors, edited by H. Haug (Academic, New York, 1988).

5J. S. Weiner, D. S. Chemla, D. A. B. Miller, T. H. Wood, D. Sivco, and A. Y. Cho, Appl. Phys. Lett. 46, 619 (1985).

${ }^{6}$ D. A. B. Miller, D. S. Chemla, P. W. Smith, A. C. Gossaard, and W. Wiegmann, Appl. Phys. B 28, 96 (1982).

${ }^{7}$ L. E. Brus, J. Chem. Phys. 79, 5566 (1983); 80, 4403 (1984).

${ }^{8}$ S. Schmitt-Rink, D. A. B. Miller, and D. S. Chemla, Phys. Rev. B 35, 8113 (1987).

${ }^{9}$ L. T. Canham, Appl. Phys. Lett. 57, 1046 (1990).

${ }^{10}$ L. T. Canham, Nature (London) 353, 335 (1991).

${ }^{11}$ V. Lehamnn and U. Gosele, Appl. Phys. Lett. 58, 856 (1991).

${ }^{12}$ S. Gardelis, J. S. Rimmer, P. Sawson, B. Hamilton, T. E. Whall, and E. H. Parker, Appl. Phys. Lett. 59, 2119 (1991).

${ }^{13}$ R. P. Vasques, R. W. Fathauer, T. George, and A. Ksendzov, Appl. Phys. Lett. 60, 1004 (1992).

${ }^{14}$ C. Tasi, K. H. Li, D. S. Kinosky, R. Z Qian, T. C. Hsu, J. T.
Irby, and S. K. Banerjee, Appl. Phys. Lett. 60, 1700 (1992).

${ }^{15}$ M. S. Brandt, D. H. Fuchs, M. Stutzmann, J. Weber, and M. Cardona, Solid State Commun. 81, 307 (1992).

${ }^{16}$ Z. Y. Zu, M. Gal, and M. Gross, Appl. Phys. Lett. 60, 1375 (1992).

${ }^{17}$ T. Ito, T. Ohta, O. Arakaki, and A. Hiraki, in Light Emission from Silicon, edited by S. S. Iyer, R. T. Collins, and L. T. Canham, MRS Symposia Proceedings No. 256 (Material Research Society, Pittsburgh, 1992), p. 127.

${ }^{18}$ E. Hanamura, Phys. Rev. B 37, 1273 (1988).

${ }^{19}$ L. Banayai and S. W. Koch, Phys. Rev. Lett. 57, 2722 (1986).

${ }^{20}$ T. Takagahara and E. Hanamura, Phys. Rev. Lett. 56, 2533 (1986).

${ }^{21}$ J. Wang, H. Jiang, W. Wang, and J. Zheng, Phys. Rev. Lett. 69, 3252 (1992).

${ }^{22}$ N. Bloembergen, Nonlinear Optics (Addison-Wesley, New York, 1991).

${ }^{23}$ The distortion of the exciton wave function caused by the confinement effect is assumed to be small in this context.

${ }^{24}$ N. Ekenberg, Phys. Rev. B 36, 6152 (1987).

${ }^{25}$ F. Buda, J. Kohanoff, and M. Parrinello, Phys. Rev. Lett. 69, 1272 (1992).

${ }^{26}$ G. D. Sanders and Y. C. Chang, Phys. Rev. B 45, 9202 (1992). 